\title{
EVOLUTIONAL METHODS FOR CREATING ARTIFICIAL INTELLIGENCE OF ROBOTIC TECHNICAL SYSTEMS
}

\author{
Askhat I. Diveev ${ }^{1}$, Sabit I. Ibadulla ${ }^{2}$ \\ ${ }^{1}$ Federal Research Center "Informatics and Management" of the Russian Academy of \\ Sciences, Peoples' Friendship University of Russia \\ phone: +7 90571144 27, e-mail: aidiveev@mail.ru \\ ${ }^{2}$ Korkyt Ata State University, Kyzylorda, Kazakhstan \\ phone: +7 77577174 00, e-mail: sabit_sss@mail.ru
}

\begin{abstract}
This paper considers evolutionary methods of symbolic regression for the creation of artificial intelligence of robotic systems. Methods of symbolic regression are reviewed and the features of their application to the solution of the problem of synthesis of control of robotic systems are indicated. The measure of the complexity of artificial intelligence is determined and the advantage of using the principle of small variations of the basic solution is shown, while creating intelligent control systems. A method of variational genetic programming is described and an example of its use for the synthesis of intellectual control is given.
\end{abstract}

Keywords: artificial intelligence, evolutionary computations, symbolic regression methods, genetic programming.

\section{INTRODUCTION}

The current problem of creating artificial intelligence with the development of robotic systems has become most acute. Despite the fact that the problem of creating artificial intelligence has been extensively discussed [1,2], the very concept of artificial intelligence and its definition are relevant. Obviously, in decision-making systems, pattern recognition, inference and learning, the presence of intelligence is necessary and, consequently, these systems developed by man must include elements of artificial intelligence.

In future, we will assume that any system created by a person that contains as an essential element the condition operator "if ..., then ..." or the "if" operator has artificial intelligence. Even a system with one "if" operator, for example, the "own - alien" pass check system, also has artificial intelligence. According to this definition, the measure of artificial intelligence is the number of necessary condition operators. In this case, it can be argued that the system "one's own" is a system with artificial intelligence of 1 "if".

The assertion that artificial intelligence is a system containing condition operators is also confirmed by the fact that it is difficult to find a mathematical apparatus in which, formally from the mathematical formulation of the problem, the condition operator is obtained as a solution. Consequently, the condition operator in the overwhelming majority of cases is a product of the developer's intelligence, which inserted the condition operator into the necessary place of the system's operation algorithm, i.e. the developer applied his/her intellect to create an artificial one.

The development of robotic systems obviously requires the creation of control systems with a large number of condition operators. It is enough to take the problem of creating an unmanned vehicle. It is easy to guess how many conditions the control system should check or how many condition operators need to be included in the intelligence of the control system so that the car can safely move around the city to the destination.

Even more complex is the control system of a group of robotic systems. When managing such a system, the problem arises of processing a large amount of information when it is necessary to know the state of all the elements of the group and, at the same time, take into

IRTIIE Vol. 6, No. 2, 2018 ISSN 1314-8788 (print), ISSN 1314-8796 (online), doi: 10.15547/artte.2018.02.010 
account that each robot is an obstacle changing its position in time for another robot from the group.

The rapid growth in the complexity of tasks that need to be addressed by systems with artificial intelligence, significantly slows down the development of these systems. The problem is further complicated by the fact that there is no formal method of creating systems with condition operators, i.e. the process of automating the development of artificial intelligence systems cannot be algorithmized without the use of search algorithms that were aimed at solving complex NP-complete problems without performing a full search of possible solutions. The development of evolutionary computations led to the creation of symbolic regression methods in which, using evolutionary algorithms, the search for optimal solutions on a space of abstract codes not containing a numerical metric, for example, program codes or mathematical expressions, is carried out.

In this paper, we consider the use of evolutionary methods of symbolic regression to create an artificial intelligence of the robotic control system.

\section{THE METHODS OF SYMBOLIC REGRESSION}

All methods of symbolic regression include two stages: the coding of the desired mathematical expression or program algorithmic recording and the search for the optimal code using an evolutionary algorithm. The first method of symbolic regression is the method of genetic programming [3]. Today, there are several methods of symbolic regression, which differ in the form of coding and the search algorithm.

Inductive genetic programming [4] uses only polynomial codes of one or two variables.

Cartesian genetic programming [5] encodes a mathematical expression by a set of computational blocks describing elementary operations.

Grammatical evolution [6] encodes expressions in the form of the universal Backus-Naur form. Analytical programming [7] encodes expressions in an ordered set of integers.

The network operator method [8] encodes a mathematical expression consisting only of functions with one or two arguments, in the form of an integer square matrix describing the structure of the oriented graph. When searching for the optimal solution, the principle of small variations of the basic solution is used.

The method of parsing matrices [9] encodes mathematically in the form of an integral nonsquare matrix consisting of vectors describing the calls of the computational blocks.

The method of variational genetic programming [10] is the application of the principle of a small basic solution to genetic programming.

The method of variational analytical programming [11] is the application of the principle of a small basic solution to analytic programming.

The multilayered network operator method [12] is designed to encode complex mathematical expressions in the form of interconnected network operators

The binary analytical programming method [13] encodes mathematical expressions consisting only of functions with one or two arguments in the form of a binary computational tree.

Using the principle of small variations of the basic solution [14] allows us to construct an abstract metric on the search space that allows us to determine the proximity of two mathematical expressions and the neighborhood of the search on the set of codes of mathematical expressions.

\section{SYNTHESIS OF AN INTELLIGENT CONTROL SYSTEM}

The methods of symbolic regression allow us to find a mathematical expression along with the logical condition operators. For example, when searching for a mobile robot control system under constraint conditions, the following multi-operator conditions were used, defined as functions with three arguments:

IRTIIE Vol. 6, No. 2, 2018 ISSN 1314-8788 (print), ISSN 1314-8796 (online), doi: 10.15547/artte.2018.02.010 


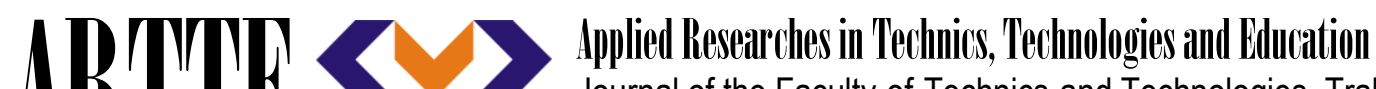 Journal of the Faculty of Technics and Technologies, Trakia University https://sites.google.com/a/trakia-uni.bg/artte/}

$$
\begin{gathered}
\mathrm{F}_{3}=\left(f_{3,0}\left(z_{1}, z_{2}, z_{3}\right)=\left\{\begin{array}{l}
z_{2}, \text { if } z_{1} \leq 0 \\
z_{3}, \text { otherwise }
\end{array}, f_{3,1}\left(z_{1}, z_{2}, z_{3}\right)=\left\{\begin{array}{l}
z_{3}, \text { if } z_{1} \leq z_{2} \\
-z_{3}, \text { otherwise }
\end{array},\right.\right.\right. \\
f_{3,2}\left(z_{1}, z_{2}, z_{3}\right)=\left\{\begin{array}{l}
z_{2}, \text { if } z_{1}<z_{2} \\
z_{3}, \text { if }\left(z_{1} \geq z_{2}\right) \wedge\left(z_{1}>z_{3}\right), \\
z_{1}, \text { if }\left(z_{1} \geq z_{2}\right) \wedge\left(z_{1} \leq z_{3}\right)
\end{array}\right. \\
f_{3,3}\left(z_{1}, z_{2}, z_{3}\right)=\left\{\begin{array}{l}
\left.z_{2}-z_{1}, \text { if }\left|z_{1}-z_{2}\right|<\left|z_{1}-z_{3}\right|\right) . \\
z_{3}-z_{1}, \text { otherwise }
\end{array}\right)
\end{gathered}
$$

The application of the method of variational analytical programming to the synthesis of control by a flying robot led to the following intelligent control system containing the condition operators:

$$
T=\left\{\begin{array}{l}
T^{+}, \text {if } \tilde{T}>T^{+} \\
T^{-}, \text {if } \tilde{T}<T^{-} \\
\tilde{T}, \text { otherwise }
\end{array} M_{z}=\left\{\begin{array}{l}
M_{z}^{+}, \text {if } \tilde{M}_{z}>M_{z}^{+} \\
M_{z}^{-}, \text {if } \tilde{M}_{z}<M_{z}^{-} \\
\tilde{M}_{z}, \text { otherwise }
\end{array} \quad M_{x}=\left\{\begin{array}{l}
M_{x}^{+}, \text {if } \tilde{M}_{x}>M_{x}^{+} \\
M_{x}^{-}, \text {if } \tilde{M}_{x}<M_{x}^{-} \\
\tilde{M}_{x}, \text { otherwise }
\end{array}\right.\right.\right.
$$

where $T$ is the pull of all four screws, $M z$ is the moment around the lateral axis, and $M x$ is the moment around the longitudinal axis.

$$
\begin{gathered}
\tilde{T}=\Delta_{x}+\sqrt[3]{\Delta_{y}}-\Delta_{x} \sqrt[3]{\Delta_{y}}, \\
\tilde{M}_{z}=\operatorname{sgn}\left(\Delta_{y}\right) e^{-\left|\Delta_{y}\right|} \operatorname{sgn}\left(\mu\left(2 \Delta_{x}\right)\right)\left(\ln \left(\left|\mu\left(2 \Delta_{x}\right)\right|+1\right)-\ln ^{3}\left(\left|\mu\left(2 \Delta_{x}\right)\right|+1\right)\right), \\
\tilde{M}_{x}=\frac{1-e^{-A}}{1+e^{-A}} \mu(a)=\left\{\begin{array}{l}
a, \text { if }|a|<1 \\
\operatorname{sgn}(a)-\text { otherwise },
\end{array}\right. \\
A=\min \left\{\operatorname{sgn}\left(\Delta_{z}\right) \ln \left(\left|\Delta_{z}\right|+1\right), \min \left\{\max \left\{\operatorname{sgn}(B) \ln (|B|), \Delta_{x}\right\}, q_{11}\right\}\right\}, \\
B=f_{3,1}\left(\ln \left(q_{12}+1\right), \Delta_{z}, \Delta_{z}\right) \ln \left(\left|f_{3,1}\left(\ln \left(q_{12}+1\right), \Delta_{z}, \Delta_{z}\right)\right|+1\right), \\
f_{3,1}(a, b, c)=\left\{\begin{array}{l}
c, \text { if } a<b \\
-c \text { otherwise }
\end{array}\right. \\
\Delta_{y}=-q_{10} \&-q_{9}\left(y-y_{i}\right)+g+\operatorname{arctg}(\&)+\sin (\&)+\left(q_{6} \theta\right)^{2}- \\
-\operatorname{sgn}\left(y-y_{i}\right) \sqrt{q_{9}\left|y-y_{i}\right|}, \\
\Delta_{x}=-q_{5} \&-q_{6} \theta+q_{7} \&+q_{8}\left(x-x_{i}\right), \\
\Delta_{z}=-q_{1} \& \&-q_{2} \gamma-q_{3} \& q_{4}\left(z-z_{i}\right),
\end{gathered}
$$

where $q_{1}=1,075, q_{2}=3, q_{3}=0,527, q_{4}=0,324, q_{5}=1,075, q_{6}=3, q_{7}=0,527$,

$$
q_{8}=0,324, q_{9}=15,51, q_{10}=15,19, q_{11}=3,234, q_{12}=1,5625 \text {. }
$$

The resulting control system provides the motion of the flying robot from different initial conditions along a given spatial trajectory (see Figure 1).

IRTIIE Vol. 6, No. 2, 2018 ISSN 1314-8788 (print), ISSN 1314-8796 (online), doi: 10.15547/artte.2018.02.010 

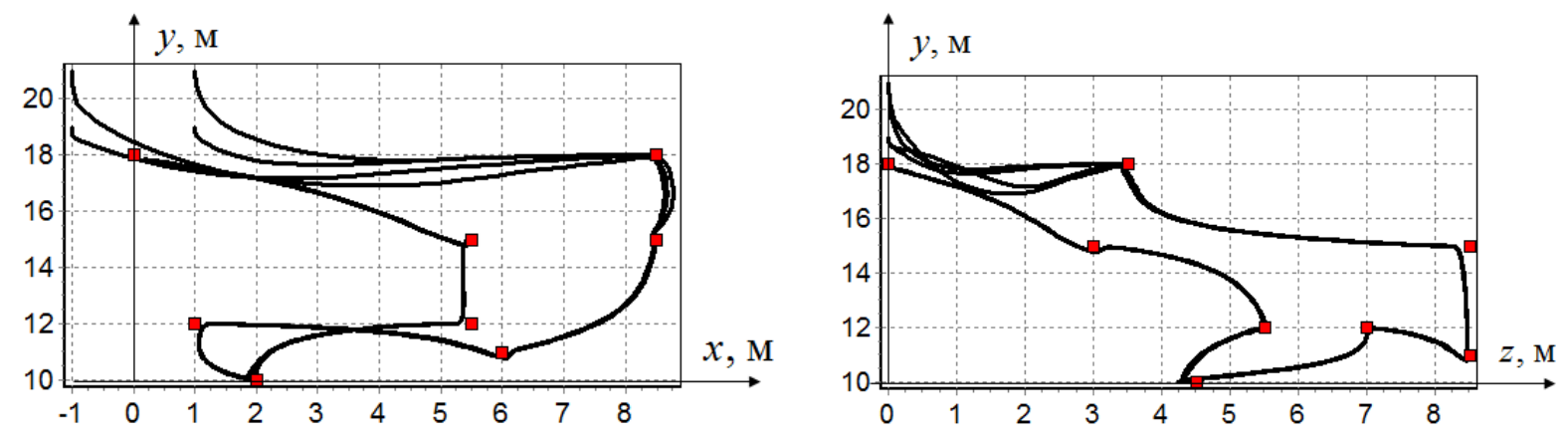

Figure 1. Movement of a flying robot along a spatial trajectory

\section{CONCLUSIONS}

Artificial intelligence systems are systems containing a condition operator. A measure of the complexity of artificial intelligence systems is the number of necessary condition operators. Using the methods of symbolic regression and evolutionary algorithms allows us to automate the process of building artificial intelligence systems and to include in the solution the necessary number of condition operators. An example of using the symbolic regression method for synthesizing control of a flying robot led to the creation of an intelligent control system that includes several condition operators.

\section{REFERENCES}

[1] Russell S., Norvig P. (2006). Artificial Intelligence. Modern approach. Moscow: Izd. house "Williams", 2006, p. 1408.

[2] Lugger J. F. (2003). Artificial Intelligence. Strategies and methods for solving complex problems. Moscow: Izd. house "Williams", 2003, p. 864.

[3] Koza J. R. (1992). Genetic Programming: On the Programming of Computers by Means of Natural Selection. Cambridge, Massachusetts, London, MA: MIT Press, 1992, p. 819.

[4] Nikolaev N., Iba H. (2000). Inductive Genetic Programming of Polynomial Learning Networks. Proc. IEEE Symposium on Combinations of Evolutionary Computation and Neural Networks ECNN-2000. X. Yao Ed. IEEE-Press, pp.158-167.

[5] Miller J., Thomson P. (2000). Cartesian Genetic Programming. Proc. EuroGP'2000R 3rd European Conf. Genetic Programming, R. Poli, W. Banzhaf, W.B. Langdon, J.F. Miller, P. Nordin, and Fogarty, T.C. Eds., Edinburgh, Scotland, vol. 1802. Berlin: SpringerVerlag, 2000, pp. 121-132.

[6] O'Neill M., Ryan C. (2001). Grammatical Evolution. IEEE Trans. Evol. Comput, 2001, 5, pp. 349-358.

[7] Zelinka I. (2002). Analytic programming by Means of SOMA Algorithm. In Proceedings of the 8th International Conference on Soft Computing Mendel 02, Brno, Czech Republic, 2002, pp. 93-101.

[8] Diveev A. I., Sofronova E. A. (2008). Proceedings of the 17th IFAC World Congress, Seoul, 2008, 05.07.2008 - 12.07.2008, pp. 6106-6113.

[9] Luo C., Zhang S.-L. (2012). Parse-matrix evolution for symbolic regression // Engineering Applications of Arti fi cial Intelligence, 2012, 25, pp. 1182-1193.

[10] Diveev A. I., Ibadulla S. I., Konyrbaev N. B., Shmalko E. Yu. (2015). Variational Genetic Programming for Optimal Control System Synthesis of Mobile Robots // Preprints of the 11th IFAC Symposium on Robot Control, Salvador, BA, Brazil, August 26-28, 2015, pp. 113-118.

IRTIIE Vol. 6, No. 2, 2018 ISSN 1314-8788 (print), ISSN 1314-8796 (online), doi: 10.15547/artte.2018.02.010 


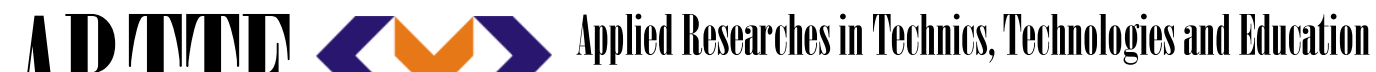 Journal of the Faculty of Technics and Technologies, Trakia University https://sites.google.com/a/trakia-uni.bg/artte/}

[11] Diveev A. I., Ibadulla S. I., Konyrbaev N. B., Shmalko E. Yu. (2015). Variational Analytic Programming for Synthesis of Optimal Control for Flying Robot // Preprints of the 11th IFAC Symposium on Robot Control, Salvador, BA, Brazil, August 26-28, 2015, pp. 8287.

[12] Diveev A. I., Shmalko E. Yu. (2016). Optimal Motion Control for Multi-Robot System by Multilayer Network Operator // ICEEA 2016, 5-7 June 2016, Hefei, China, pp. 2164 2169.

[13] Diveev A. I., Konyrbaev N. B., Sofronova E. A.. (2017). INTROS'16, 5-7 October 2016, Moscow, Russia, Procedures Computer Science 103 (2017) 597-604. Method of binary analytic programming to look for optimal mathematical expression. XIIth International Symposium.

[14] Diveev A. I. (2015). Small Variations of the Basic Solution Method for Non-numerical Optimization // Proceedings of the 16th IFAC Workshop on Control Applications of Optimization, October 6th- 9th 2015 Garmisch-Partenkirchen, pp. 28-33. 